\title{
An efficient finite element method and error analysis for eigenvalue problem of Schrödinger equation with an inverse square potential on spherical domain
}

\author{
Yubing Sui ${ }^{\dagger}$, Donghao Zhang ${ }^{2 \dagger}$, Junying $\mathrm{CaO}^{3 *}$ and Jun Zhang ${ }^{4 \dagger}$
}

\author{
"Correspondence: \\ caojunying@gzmu.edu.cn \\ ${ }^{3}$ School of Data Science and \\ Information Engineering, Guizhou \\ Minzu University, 550025 Guiyang, \\ China \\ Full list of author information is \\ available at the end of the article \\ ${ }^{\dagger}$ Equal contributors
}

\begin{abstract}
We provide an effective finite element method to solve the Schrödinger eigenvalue problem with an inverse potential on a spherical domain. To overcome the difficulties caused by the singularities of coefficients, we introduce spherical coordinate transformation and transfer the singularities from the interior of the domain to its boundary. Then by using orthogonal properties of spherical harmonic functions and variable separation technique we transform the original problem into a series of one-dimensional eigenvalue problems. We further introduce some suitable Sobolev spaces and derive the weak form and an efficient discrete scheme. Combining with the spectral theory of Babuška and Osborn for self-adjoint positive definite eigenvalue problems, we obtain error estimates of approximation eigenvalues and eigenvectors. Finally, we provide some numerical examples to show the efficiency and accuracy of the algorithm.
\end{abstract}

MSC: $65 \mathrm{~N} 15 ; 65 \mathrm{~N} 30$

Keywords: Eigenvalue problem; Singularity; Dimension reduction scheme; Finite element method; Error estimation

\section{Introduction}

The Schrödinger eigenvalue problem with the inverse-square (IS) or centrifugal potential is widely used in nuclear physics, quantum physics, nonlinear optics, and so on [1-6]. The potential in many electronic equations produces singularity and can describe the attraction or repulsion between objects, which usually leads to strong singularities of the eigenfunctions, and this cannot be simply regarded as a perturbation term [7-11]. Therefore new tools and techniques, different from linear elliptic operators with bounded coefficients, are urgently needed for such an eigenvalue problem with the IS potential, both in analysis and in numerics. Ghanbari et al. [12-15] and Khater et al. [16-20] discussed some effective numerical methods to remove the singularity of nonlocal operators. In addition, some other works [21-26] mainly focus on studying exact solitary wave solutions.

(c) The Author(s) 2020. This article is licensed under a Creative Commons Attribution 4.0 International License, which permits use sharing, adaptation, distribution and reproduction in any medium or format, as long as you give appropriate credit to the original author(s) and the source, provide a link to the Creative Commons licence, and indicate if changes were made. The images or other third party material in this article are included in the article's Creative Commons licence, unless indicated otherwise in a credit line to the material. If material is not included in the article's Creative Commons licence and your intended use is not permitted by statutory regulation or exceeds the permitted use, you will need to obtain permission directly from the copyright holder. To view a copy of this licence, visit http://creativecommons.org/licenses/by/4.0/. 
In the last decade, increasing attention is paid to the numerical approximation of Schrödinger operators with similar singular potentials [1, 27-30]. If we solve the Schrödinger eigenvalue problem directly in a three-dimensional domain, it will take a lot of computing time and memory capacity to obtain high-precision numerical solutions $[11,31,32]$. In practice, we usually need to solve the Schrödinger eigenvalue problem on a three-dimensional spherical domain. As far as we know, there is little work discussing the eigenvalue problem with IS potential on a spherical domain.

The purpose of this work is developing an effective finite element method for the eigenvalue problem of the Schrödinger equation with an IS potential on spherical domain. To overcome the difficulties caused by the singularities of coefficients, we introduce spherical coordinate transformation and transfer the singularities from the interior of the domain to the boundary of the domain. Then by using orthogonal properties of spherical harmonic functions and variable separation technique we reduce the original problem to a series of one-dimensional eigenvalue problems. We further introduce some suitable Sobolev spaces and derive an effective discrete scheme. Combined with the theory of self-adjoint eigenvalues, we obtain error estimates of approximation eigenvalues and eigenvectors. Finally, we give several numerical examples to verify the convergence and accuracy of the algorithm.

The rest of the paper is organized as follows. In Sect. 2, we obtain a dimensional reduction scheme for the Schrödinger eigenvalue problem with IS potential. In Sect. 3, we construct a weak form and numerical discretization scheme. In Sect. 4, we obtain error estimates of approximation eigenvalues and eigenfunctions. In Sect. 5, we study the implementation details of the algorithm. In Sect. 6, we test the accuracy and convergence of the numerical algorithm. Finally, in Sect. 7, we provide some concluding remarks.

\section{Dimension reduction scheme}

In this paper, we consider the following eigenvalue problem with Dirichlet boundary conditions:

$$
\begin{aligned}
& -\Delta u+\frac{c^{2}}{x^{2}+y^{2}+z^{2}} u=\lambda u \quad \text { in } \Omega, \\
& u=0 \quad \text { on } \partial \Omega
\end{aligned}
$$

where $c$ is a bounded constant, and $\Omega=\left\{(x, y, z) \in \mathbb{R}^{3}: 0 \leq a<r<b\right\}$ with $r=\sqrt{x^{2}+y^{2}+z^{2}}$. Let $x=r \sin \theta \cos \phi, y=r \sin \theta \sin \phi, z=r \cos \theta, \psi(r, \theta, \phi)=u(x, y, z)$. We take the Laplacian in spherical coordinates

$$
\mathcal{L} \psi=\frac{1}{r^{2}} \frac{\partial}{\partial r}\left(r^{2} \frac{\partial \psi}{\partial r}\right)+\frac{1}{r^{2}} \Delta_{S} \psi
$$

where

$$
\Delta_{S}=\frac{1}{\sin \theta} \frac{\partial}{\partial \theta}\left(\sin \theta \frac{\partial}{\partial \theta}\right)+\frac{1}{\sin ^{2} \theta} \frac{\partial^{2}}{\partial \phi^{2}} .
$$


Then (1)-(2) can be rewritten as the eigenvalue problem of the Schrödinger equation in spherical coordinates:

$$
\begin{aligned}
& -\mathcal{L} \psi+\frac{c^{2}}{r^{2}} \psi=\lambda \psi, \quad(r, \theta, \phi) \in(a, b) \times[0, \pi] \times[0,2 \pi], \\
& \text { (1) } \psi(b, \theta, \phi)=0, \quad(\theta, \phi) \in[0, \pi] \times[0,2 \pi] \text { for } a=0, \\
& \text { (2) } \psi(a, \theta, \phi)=\varphi(b, \theta, \phi)=0, \quad(\theta, \phi) \in[0, \pi] \times[0,2 \pi] \text { for } a>0 .
\end{aligned}
$$

We recall that an important property of the spherical harmonics $\left\{Y_{m}^{l}\right\}$ (as normalized in [33]) is that they are eigenfunctions of the Laplace-Beltrami operator $\Delta_{S}$. More precisely,

$$
\Delta_{S} Y_{l}^{m}=-l(l+1) Y_{l}^{m}, \quad l \geq 0,|m| \leq l,
$$

and from the definition of $L^{2}(S)$ we find

$$
\int_{S} Y_{l}^{m} Y_{l^{\prime}}^{m^{\prime}} d S=\delta_{l l^{\prime}} \delta_{m m^{\prime}}
$$

Using spherical harmonic expansion, we obtain that

$$
\psi(r, \theta, \phi)=\sum_{l=0}^{\infty} \sum_{|m|=0}^{l} \psi_{l}^{m} Y_{l}^{m} .
$$

First of all, we take into account the case $a=0$. Define the differential operator $\tilde{L}_{l} \psi_{l}^{m}=$ $\frac{1}{r^{2}}\left(\partial_{r}\left(r^{2} \partial_{r} \psi_{l}^{m}\right)-l(l+1) \psi_{l}^{m}\right)$. By substituting expression (10) into (5) and (6) we can obtain a series of equivalent one-dimensional eigenvalue problems:

$$
\begin{aligned}
& -\tilde{L}_{l} \psi_{l}^{m}+\frac{c^{2}}{r^{2}} \psi_{l}^{m}=\lambda_{l} \psi_{l}^{m}, \quad r \in(0, b), \\
& \psi_{l}^{m}(b)=0 .
\end{aligned}
$$

Let $r=\frac{t+1}{2} b, u_{l}^{m}(t)=\psi_{l}^{m}\left(\frac{t+1}{2} b\right)$, and $\mathcal{L}_{l} u_{l}^{m}=\frac{1}{(t+1)^{2}} \partial_{t}\left((t+1)^{2} \partial_{t} u_{l}^{m}\right)-\frac{l(l+1)}{(t+1)^{2}} u_{l}^{m}$. Then (11)(12) can be rewritten as

$$
\begin{aligned}
& -\mathcal{L}_{l} u_{l}^{m}+\frac{c^{2}}{(t+1)^{2}} u_{l}^{m}=\frac{b^{2}}{4} \lambda_{l} u_{l}^{m}, \quad t \in(-1,1), \\
& u_{l}^{m}(1)=0 .
\end{aligned}
$$

Analogously, for the case $a>0$, inserting expression (10) into (5) and (7), we can derive the following one-dimensional eigenvalue problem:

$$
\begin{aligned}
& -\tilde{L}_{l} \psi_{l}^{m}+\frac{c^{2}}{r^{2}} \psi_{l}^{m}=\lambda_{l} \psi_{l}^{m}, \quad r \in(0, b), \\
& \psi_{l}^{m}(a)=\psi_{l}^{m}(b)=0 .
\end{aligned}
$$


Let $r=\frac{b-a}{2} t+\frac{b+a}{2}, u_{l}^{m}(t)=\psi_{l}^{m}(r)$, and $L_{l} u_{l}^{m}=\frac{4}{(b-a)^{2} r^{2}} \partial_{t}\left(r^{2} \partial_{t} u_{l}^{m}\right)-\frac{l(l+1)}{r^{2}} u_{l}^{m}$. From (15)-(16) we derive that

$$
\begin{aligned}
& -\mathcal{L}_{l} u_{l}^{m}+\frac{c^{2}}{r^{2}} u_{l}^{m}=\lambda_{l} u_{l}^{m}, \quad t \in(-1,1), \\
& u_{l}^{m}(-1)=u_{l}^{m}(1)=0 .
\end{aligned}
$$

\section{Weak form and discrete scheme}

When $a=0$, we can define the space

$$
H_{0, l}^{1}(I):=\left\{u_{l}^{m}: \int_{I}(t+1)^{2}\left|\partial_{t} u_{l}^{m}\right|^{2}+\left[l(l+1)+c^{2}\right] u^{2} d t<\infty, u_{l}^{m}(1)=0\right\} .
$$

The inner product and norm can be defined as follows:

$$
\begin{aligned}
& \left(u_{l}^{m}, v_{l}^{m}\right)_{1, l}=\int_{I}(t+1)^{2} \partial_{t} u_{l}^{m} \partial_{t} v_{l}^{m}+\left[l(l+1)+c^{2}\right] u_{l}^{m} v_{l}^{m} d t \\
& \left\|u_{l}^{m}\right\|_{1, l}=\left(u_{l}^{m}, u_{l}^{m}\right)_{1, l}^{\frac{1}{2}}
\end{aligned}
$$

Thus the weak form of (13)-(14) is: Find $\left(\lambda_{l}, u_{l}^{m}\right) \in \mathbb{R} \times H_{0, l}^{1}(I)$ such that

$$
a_{l}\left(u_{l}^{m}, v_{l}^{m}\right)=\lambda_{l} b_{l}\left(u_{l}^{m}, v_{l}^{m}\right), \quad \forall v_{l}^{m} \in H_{0, l}^{1}(I)
$$

where

$$
\begin{aligned}
& a_{l}\left(u_{l}^{m}, v_{l}^{m}\right)=\int_{I}(t+1)^{2} \partial_{t} u_{l}^{m} \partial_{t} v_{l}^{m}+\left[l(l+1)+c^{2}\right] u_{l}^{m} v_{l}^{m} d t \\
& b_{l}\left(u_{l}^{m}, v_{l}^{m}\right)=\frac{b^{2}}{4} \int_{I}(t+1)^{2} u_{l}^{m} v_{l}^{m} d t .
\end{aligned}
$$

We denote by $\tilde{V}_{h}(l)=\tilde{P}_{1 h} \cap H_{0, l}^{1}(I)$ the approximation space, where $\tilde{P}_{1 h}$ is a piecewise linear interpolation polynomial space. Thus the corresponding numerical scheme of (19) is: Find $\left(\lambda_{l h}, u_{l h}^{m}\right) \in \mathbb{R} \times \tilde{V}_{h}(l)$ such that

$$
a_{l}\left(u_{l h}^{m}, v_{l h}^{m}\right)=\lambda_{l h} b_{l}\left(u_{l h}^{m}, v_{l h}^{m}\right), \quad \forall v_{l h}^{m} \in \tilde{V}_{h}(l) .
$$

When $a>0$, the usual space can be denoted as

$$
H_{0}^{1}(I):=\left\{u_{l}^{m}: u_{l}^{m}, \partial_{t} u_{l}^{m} \in L^{2}(I), u_{l}^{m}(-1)=u_{l}^{m}(1)=0\right\} .
$$

We define the inner product and norm as

$$
\left(u_{l}^{m}, v_{l}^{m}\right)_{1, I}=\int_{I}\left(\partial_{t} u_{l}^{m} \partial_{t} v_{l}^{m}+u_{l}^{m} v_{l}^{m}\right) d t, \quad\left\|u_{l}^{m}\right\|_{1, I}=\left(u_{l}^{m}, u_{l}^{m}\right)_{1, I^{\frac{1}{2}}}^{\frac{1}{2}}
$$

Thus the weak form of (17)-(18) is: Find $\left(\lambda_{l}, u_{l}^{m}\right) \in \mathbb{R} \times H_{0}^{1}(I)$ such that

$$
a_{l}\left(u_{l}^{m}, v_{l}^{m}\right)=\lambda_{l} b_{l}\left(u_{l}^{m}, v_{l}^{m}\right), \quad \forall v_{l}^{m} \in H_{0}^{1}(I)
$$


where

$$
\begin{aligned}
& a_{l}\left(u_{l}^{m}, v_{l}^{m}\right)=\int_{I} \frac{4}{(b-a)^{2}} r^{2} \partial_{t} u_{l}^{m} \partial_{t} v_{l}^{m}+\left[l(l+1)+c^{2}\right] u_{l}^{m} v_{l}^{m} d t \\
& b_{l}\left(u_{l}^{m}, v_{l}^{m}\right)=\int_{I} r^{2} u_{l}^{m} v_{l}^{m} d t .
\end{aligned}
$$

We denote by $V_{h}=P_{1 h} \cap H_{0}^{1}(I)$ the approximation space, where $P_{1 h}$ is a piecewise linear interpolation polynomial space. Then the corresponding numerical scheme of (21) is: Find $\left(\lambda_{l h}, u_{l h}^{m}\right) \in \mathbb{R} \times S_{h}(l)$ such that

$$
a_{l}\left(u_{l h}^{m}, v_{l h}^{m}\right)=\lambda_{l h} b_{l}\left(u_{l h}^{m}, v_{l h}^{m}\right), \quad \forall v_{l h}^{m} \in V_{h} .
$$

\section{Error estimation of approximation solutions}

In this section, we prove error estimates of approximate eigenvalues and eigenfunctions. Without loss of generality, we only consider the case $a>0$.

Use the technique of [34], we deduce the following results.

Theorem $1 a_{l}\left(u_{l}^{m}, v_{l}^{m}\right)$ is positive definite and continuous on $H_{0}^{1}(I) \times H_{0}^{1}(I)$, that is,

$$
\begin{aligned}
& \left|a_{l}\left(u_{l}^{m}, v_{l}^{m}\right)\right| \lesssim\left\|u_{l}^{m}\right\|_{1, I}\left\|v_{l}^{m}\right\|_{1, I}, \\
& a_{l}\left(u_{l}^{m}, u_{l}^{m}\right) \gtrsim\left\|u_{l}^{m}\right\|_{1, I}^{2}
\end{aligned}
$$

Proof Using the Cauchy-Schwarz inequality, we find

$$
\begin{aligned}
\left|a_{l}\left(u_{l}^{m}, v_{l}^{m}\right)\right| & =\left|\int_{I} \frac{4}{(b-a)^{2}} r^{2} \partial_{t} u_{l}^{m} \partial_{t} v_{l}^{m}+\left[l(l+1)+c^{2}\right] u_{l}^{m} v_{l}^{m} d t\right| \\
& \leq \int_{I} \frac{4}{(b-a)^{2}} b^{2}\left|\partial_{t} u_{l}^{m} \partial_{t} v_{l}^{m}\right|+\left[l(l+1)+c^{2}\right]\left|u_{l}^{m} v_{l}^{m}\right| d t \\
& \lesssim \int_{I}\left|\partial_{t} u_{l}^{m} \partial_{t} v_{l}^{m}\right|+\left|u_{l}^{m} v_{l}^{m}\right| d t \\
& \leq\left(\int_{I}\left|\partial_{t} u_{l}^{m}\right|^{2} d t\right)^{\frac{1}{2}}\left(\int_{I}\left|\partial_{t} v_{l}^{m}\right|^{2} d t\right)^{\frac{1}{2}}+\left(\int_{I}\left|u_{l}^{m}\right|^{2} d t\right)^{\frac{1}{2}}\left(\int_{I}\left|v_{l}^{m}\right|^{2} d t\right)^{\frac{1}{2}} \\
& \leq\left[\int_{I}\left|\partial_{t} u_{l}^{m}\right|^{2}+\left|u_{l}^{m}\right|^{2} d t\right]^{\frac{1}{2}}\left[\int_{I}\left|\partial_{t} v_{l}^{m}\right|^{2}+\left|v_{l}^{m}\right|^{2} d t\right]^{\frac{1}{2}} \\
& =\left\|u_{l}^{m}\right\|_{1, I}\left\|v_{l}^{m}\right\|_{1, I} .
\end{aligned}
$$

From the Poincaré inequality we derive that

$$
\begin{aligned}
a_{l}\left(u_{l}^{m}, u_{l}^{m}\right) & =\int_{I} \frac{4}{(b-a)^{2}} r^{2}\left(\partial_{t} u_{l}^{m}\right)^{2}+\left[l(l+1)+c^{2}\right]\left(u_{l}^{m}\right)^{2} d t \\
& \geq \int_{I} \frac{4}{(b-a)^{2}} a^{2}\left(\partial_{t} u_{l}^{m}\right)^{2} d t \gtrsim\left|u_{l}^{m}\right|_{1, I}^{2} \gtrsim\left\|u_{l}^{m}\right\|_{1, I^{\prime}}^{2}
\end{aligned}
$$

Similarly, we have the following conclusions. 
Theorem $2 b_{l}\left(u_{l}^{m}, v_{l}^{m}\right)$ is also positive definite and continuous on $L^{2}(I) \times L^{2}(I)$, that is,

$$
\begin{aligned}
& \left|b_{l}\left(u_{l}^{m}, v_{l}^{m}\right)\right| \lesssim\left\|u_{l}^{m}\right\|_{0, I}\left\|v_{l}^{m}\right\|_{0, I^{\prime}}, \\
& b_{l}\left(u_{l}^{m}, u_{l}^{m}\right) \gtrsim\left\|u_{l}^{m}\right\|_{0, I}^{2} .
\end{aligned}
$$

Let $V\left(\lambda_{l}\right)$ and $V\left(\lambda_{l h}\right)$ be the eigenfunction spaces corresponding to the eigenvalues $\lambda_{l}$ and $\lambda_{l h}$, respectively. Let

$$
\varepsilon_{h}=\sup _{u_{l}^{m} \in V\left(\lambda_{l}\right),\left\|u_{l}^{m}\right\|_{a_{l}}=1} \inf _{l h} \in V_{h}\left\|u_{l}^{m}-v_{l h}^{m}\right\|_{a_{l}}
$$

where $\left\|u_{l}^{m}\right\|_{a_{l}}=\sqrt{a_{l}\left(u_{l}^{m}, u_{l}^{m}\right)}$. From Theorems 1 and 2 we know that $a_{l}\left(u_{l}^{m}, v_{l}^{m}\right)$ (resp., $\left.b_{l}\left(u_{l}^{m}, v_{l}^{m}\right)\right)$ is a symmetric, continuous, and coercive bilinear form on $H_{0}^{1}(I) \times H_{0}^{1}(I)$ (resp., $\left.L^{2}(I) \times L^{2}(I)\right)$.

By the spectral theory of eigenvalue problems [34] we have the following theorem.

Theorem 3 Let $\left(\lambda_{l}, u_{l}^{m}\right)$ and $\left(\lambda_{l h}, u_{l h}^{m}\right)$ be respectively the eigenpairs of (21) and (22). Then the following inequalities hold:

$$
\begin{aligned}
& \left\|u_{l}^{m}-u_{l h}^{m}\right\|_{a_{l}} \lesssim \varepsilon_{h}, \\
& \lambda_{l h}-\lambda_{l} \lesssim \varepsilon_{h}^{2} .
\end{aligned}
$$

Define the piecewise linear interpolation operator $I_{h}: H_{0}^{1}(I) \rightarrow V_{h}$ by

$$
I_{h} u_{l}^{m}(t)=p_{l i}(t), \quad t \in I_{i}
$$

where $p_{l i}(t)$ denotes the linear interpolation polynomial of $u_{l}^{m}$ on the interval $I_{i}=\left[t_{i-1}, t_{i}\right]$. Let

$$
u_{l i}^{m}(t)=u_{l}^{m}(t), \quad t \in I_{i} .
$$

Then from an error formula of linear interpolating remainder term we derive that

$$
u_{l i}^{m}(t)-p_{l i}(t)=\frac{\left(u_{l i}^{m}\right)^{(2)}\left(\xi_{i}(t)\right)}{2 !}\left(t-t_{i-1}\right)\left(t-t_{i}\right)
$$

where $\xi_{i}(t) \in I_{i}$ is a function depending on $t$.

For $u_{l}^{m}$, we have the following error results.

Theorem 4 Let $K_{l i}(t)=\frac{\left(u_{l i}^{m}\right)^{(2)}\left(\xi_{i}(t)\right)}{2 !}, u_{l}^{m} \in H_{0}^{1}(I)$. Suppose that $u_{l}^{m}$ is smooth enough such that $\left|\partial_{t}^{k} K_{l i}(t)\right| \leq M(k=0,1)$, where $M$ is a positive constant. Then

$$
\left|I_{h} u_{l}^{m}-u_{l}^{m}\right|_{1, I} \lesssim h
$$

where $h=\max _{1 \leq i \leq n}\left\{h_{i}\right\}, h_{i}=t_{i}-t_{i-1}$. 
Proof Since

$$
u_{l i}^{m}(t)-p_{l i}(t)=K_{l i}(t)\left(t-t_{i-1}\right)\left(t-t_{i}\right)
$$

we have

$$
\partial_{t}\left(u_{l i}^{m}(t)-p_{l i}(t)\right)=\partial_{t} K_{l i}(t)\left(t-t_{i-1}\right)\left(t-t_{i}\right)+K_{l i}(t) \partial_{t}\left(\left(t-t_{i-1}\right)\left(t-t_{i}\right)\right)
$$

Thus we obtain

$$
\begin{aligned}
\left|\partial_{t}\left(u_{l i}^{m}(t)-p_{l i}(t)\right)\right|^{2} & \lesssim\left|\left(t-t_{i-1}\right)\left(t-t_{i}\right)\right|^{2}+\left|\partial_{t}\left(\left(t-t_{i-1}\right)\left(t-t_{i}\right)\right)\right|^{2} \\
& =\left|\left(t-t_{i-1}\right)\left(t-t_{i}\right)\right|^{2}+\left|\left(t-t_{i}\right)+\left(t-t_{i-1}\right)\right|^{2} \\
& \leq\left(\frac{h_{i}}{2}\right)^{4}+\left(2 h_{i}\right)^{2} \lesssim h_{i}^{2} .
\end{aligned}
$$

From the Poincaré inequality we derive that

$$
\begin{aligned}
\left\|I_{h} u_{l}^{m}-u_{l}^{m}\right\|_{1, I}^{2} & \lesssim\left|I_{h} u_{l}^{m}-u_{l}^{m}\right|_{1, I}^{2} \\
& =\sum_{i=1}^{n}\left|I_{h} u_{l}^{m}-u_{l}^{m}\right|_{1, I_{i}}^{2}=\sum_{i=1}^{n}\left|p_{l i}-u_{l i}^{m}\right|_{1, I_{i}}^{2} \\
& =\sum_{i=1}^{n} \int_{I_{i}}\left|\partial_{t}\left(u_{l i}^{m}(t)-p_{l i}(t)\right)\right|^{2} d t \\
& \lesssim \sum_{i=1}^{n} h_{i}^{3} \lesssim h^{2} .
\end{aligned}
$$

This ends the proof.

We can get the following standard error estimation results.

Theorem 5 Let $\left(\lambda_{l}, u_{l}^{m}\right)$ and $\left(\lambda_{l h}, u_{l h}^{m}\right)$ be the eigenpairs of (21) and (22), respectively. If $u_{l}^{m} \in H_{0}^{1}(I)$ satisfy the condition of Theorem 4, then the following inequalities hold:

$$
\left\|u_{l}^{m}-u_{l h}^{m}\right\|_{a_{l}} \lesssim h, \quad \lambda_{l h}-\lambda_{l} \lesssim h^{2}
$$

Proof Since

$$
\begin{aligned}
\varepsilon_{h} & =\sup _{u_{l}^{m} \in V\left(\lambda_{l}\right),\left\|u_{l}^{m}\right\|_{a_{l}}=1} \inf _{v_{l h}^{m} \in V_{h}}\left\|u_{l}^{m}-v_{l h}^{m}\right\|_{a_{l}} \\
& \leq \sup _{u_{l}^{m} \in V\left(\lambda_{l}\right),\left\|u_{l}^{m}\right\|_{a_{l}=1}}\left\|u_{l}^{m}-I_{h} u_{l h}^{m}\right\|_{a_{l}} \\
& \lesssim \sup _{u_{l}^{m} \in V\left(\lambda_{l}\right),\left\|u_{l}^{m}\right\|_{a_{l}=1}}\left\|u_{l}^{m}-I_{h} u_{l h}^{m}\right\|_{1, I^{\prime}}
\end{aligned}
$$


from the Poincaré inequality and Theorem 4 we derive that

$$
\begin{aligned}
\varepsilon_{h} & \lesssim \sup _{u_{l}^{m} \in V\left(\lambda_{l}\right),\left\|u_{l}^{m}\right\|_{a_{l}}=1}\left\|u_{l}^{m}-I_{h} u_{l h}^{m}\right\|_{1, I} \\
& \lesssim \sup _{u_{l}^{m} \in V\left(\lambda_{l}\right),\left\|u_{l}^{m}\right\|_{a_{l}=1}}\left|u_{l}^{m}-I_{h} u_{l h}^{m}\right|_{1, I} \\
& \lesssim h .
\end{aligned}
$$

Combining this with Theorem 3, we obtain (28).

\section{Implementation of the numerical scheme}

In this section, we present the algorithm to solve problems (20) and (22). First, we define some basis functions. Let

$$
\begin{aligned}
& \psi_{0}(t)= \begin{cases}-\frac{t-t_{1}}{h_{1}}, & t_{0} \leq t \leq t_{1}, \\
0, & \text { otherwise }\end{cases} \\
& \psi_{i}(t)= \begin{cases}\frac{t-t_{i-1}}{h_{i}}, & t_{i-1} \leq t \leq t_{i}, \\
-\frac{t-t_{i+1}}{h_{i+1}}, & t_{i} \leq t \leq t_{i+1}, \\
0, & \text { otherwise }\end{cases}
\end{aligned}
$$

where $i=1, \ldots, N-1$. We find that

$$
\begin{gathered}
\tilde{V}_{h}=\operatorname{span}\left\{\psi_{0}(t), \ldots, \psi_{N-1}(t)\right\}, \\
V_{h}=\operatorname{span}\left\{\psi_{1}(t), \ldots, \psi_{N-1}(t)\right\} .
\end{gathered}
$$

Case 1. $a=0$. Set

$$
\begin{aligned}
& a_{i j}=\int_{I}(t+1)^{2}\left(\psi_{j}\right)^{\prime}\left(\psi_{i}\right)^{\prime} d t, \quad b_{i j}=\int_{I} \psi_{j} \psi_{i} d t, \\
& c_{i j}=\int_{I}(t+1)^{2} \psi_{j} \psi_{i} d t .
\end{aligned}
$$

Let

$$
u_{l h}^{m}=\sum_{i=0}^{N-1} u_{i} \psi_{i} .
$$

Substituting expression (29) into (20) and noticing the $v_{l h}^{m}$, we can observe the linear system

$$
\left[\mathbb{A}+\left(l^{2}+l+c^{2}\right) \mathbb{B}\right] \mathbb{U}=\frac{b^{2}}{4} \lambda_{l h} \mathbb{C} \mathbb{U}
$$

where

$$
\mathbb{A}=\left(a_{i j}\right), \quad \mathbb{B}=\left(b_{i j}\right), \quad \mathbb{C}=\left(c_{i j}\right), \quad \mathbb{U}=\left(u_{0}, \ldots, u_{N-1}\right)^{T} .
$$


From the properties of the basis functions we know that the stiff matrices and mass matrix in (30) are all tridiagonal sparse matrices.

Case 2. $a>0$. Let

$$
u_{l h}^{m}=\sum_{i=1}^{N-1} u_{i} \psi_{i} .
$$

Substituting expression (31) into (22) and taking $v_{l h}^{m}$ in $V_{h}$, we obtain the linear system

$$
\mathcal{A U}=\lambda_{l h} \mathcal{B U}
$$

where

$$
\begin{aligned}
& \mathcal{A}=\left(a_{i j, l}\right), \quad \mathcal{B}=\left(b_{i j, l}\right), \quad a_{i j, l}=a_{l}\left(\psi_{j}, \psi_{i}\right), \quad b_{i j, l}=b_{l}\left(\psi_{j}, \psi_{i}\right), \\
& \mathcal{U}=\left(u_{1}, \ldots, u_{N-1}\right)^{T} .
\end{aligned}
$$

Similarly, from the properties of the basis functions we know that the stiff matrices and mass matrix in (32) are all tridiagonal sparse matrices.

Remark 1 Our numerical method can transform three-dimensional problems into a series of eigenvalue problems. By constructing appropriate basis functions these onedimensional value problems will be discretized into asparse stiffness matrix and mass matrix, which can be efficiently solved.

\section{Numerical experiments}

In this section, we present several numerical examples to check the convergence and accuracy of the numerical algorithm.

Example 1 We take $c=0, a=0, b=1$, and $l=0,1,2$ as our example. In Tables $1-3$, we provide the first four eigenvalues with different $l$ and $h$ of Example 1.

Example 2 We take $c=\frac{1}{3}, a=0, b=1$, and $l=0,1,2$ as our example. In Tables $1-3$, we give the first four eigenvalues with different $l$ and $h$ of Example 2.

We know from Tables 1-6 that the approximation eigenvalues achieve at least threedigit accuracy with $h \leq \frac{1}{512}$ for $l=0,1,2$. To further show the convergence of approximation eigenvalues, we let the numerical solution of $h=\frac{1}{1024}$ be the reference solution. The error of the approximate eigenvalues with different $h$ are provided in Figs. 1-6.

We observe from Figs. 1-6 that the numerical eigenvalues are convergent.

Table $1 a=0$, the four eigenvalues with $/=0$ and $h$

\begin{tabular}{lllll}
\hline$h$ & $\lambda_{0 h}^{1}$ & $\lambda_{0 h}^{2}$ & $\lambda_{0 h}^{3}$ & $\lambda_{0 h}^{4}$ \\
\hline $1 / 64$ & 9.869857717467 & 39.497938355087 & 88.947662545164 & 158.330138143300 \\
$1 / 128$ & 9.869667769569 & 39.483298848912 & 88.856747023505 & 158.017765253015 \\
$1 / 256$ & 9.869620245684 & 39.479637982214 & 88.834016573723 & 157.939692761527 \\
$1 / 512$ & 9.869608362394 & 39.478722703012 & 88.8283333857921 & 157.920175919198 \\
$1 / 1024$ & 9.869605391441 & 39.478493879297 & 88.826913172297 & 157.915296787651 \\
\hline
\end{tabular}


Table $2 a=0$, the four eigenvalues with $/=1$ and $h$

\begin{tabular}{lllll}
\hline$h$ & $\lambda_{1 h}^{1}$ & $\lambda_{1 h}^{2}$ & $\lambda_{1 h}^{3}$ & $\lambda_{1 h}^{4}$ \\
\hline $1 / 64$ & 20.192597755854 & 59.718503283484 & 119.092115617980 & 198.448082264746 \\
$1 / 128$ & 20.191196002143 & 59.689264430485 & 118.947930249028 & 198.005334602179 \\
$1 / 256$ & 20.190845426967 & 59.681953168655 & 118.911884395986 & 197.894689220523 \\
$1 / 512$ & 20.190757774611 & 59.680125256653 & 118.902872969163 & 197.867030522764 \\
$1 / 1024$ & 20.190735860985 & 59.679668272588 & 118.900620114823 & 197.860116014585 \\
\hline
\end{tabular}

Table $3 a=0$, the four eigenvalues with $/=2$ and $h$

\begin{tabular}{lllll}
\hline$h$ & $\lambda_{2 h}^{1}$ & $\lambda_{2 h}^{2}$ & $\lambda_{2 h}^{3}$ & $\lambda_{2 h}^{4}$ \\
\hline $1 / 64$ & 33.221527912004 & 82.779965293070 & 152.120849702143 & 241.466504738343 \\
$1 / 128$ & 33.218478774072 & 82.734418406613 & 151.921374315241 & 240.893774149263 \\
$1 / 256$ & 33.217716151749 & 82.723028162111 & 151.871499583334 & 240.750621394948 \\
$1 / 512$ & 33.217525475045 & 82.720180381294 & 151.859030542575 & 240.714835156415 \\
$1 / 1024$ & 33.217477804567 & 82.719468422366 & 151.855913260174 & 240.705888719943 \\
\hline
\end{tabular}

Table $4 a=0$, the four eigenvalues with $I=0$ and $h$

\begin{tabular}{lllll}
\hline$h$ & $\lambda_{0 h}^{1}$ & $\lambda_{0 h}^{2}$ & $\lambda_{0 h}^{3}$ & $\lambda_{0 h}^{4}$ \\
\hline $1 / 64$ & 10.784422087149 & 41.409061237094 & 91.862254368608 & 162.255452978584 \\
$1 / 128$ & 10.783897714384 & 41.391953774531 & 91.763361765200 & 161.924692186926 \\
$1 / 256$ & 10.783722018000 & 41.387479496580 & 91.738161486718 & 161.841125052992 \\
$1 / 512$ & 10.783658754253 & 41.386276025201 & 91.731657235236 & 161.819852309708 \\
$1 / 1024$ & 10.783634535429 & 41.385938328125 & 91.729942732668 & 161.814368929169 \\
\hline
\end{tabular}

Table $5 a=0$, the four eigenvalues with $/=1$ and $h$

\begin{tabular}{lllll}
\hline$h$ & $\lambda_{1 h}^{1}$ & $\lambda_{1 h}^{2}$ & $\lambda_{1 h}^{3}$ & $\lambda_{1 h}^{4}$ \\
\hline $1 / 64$ & 20.622531429872 & 60.512755868097 & 120.249994940413 & 199.970863052496 \\
$1 / 128$ & 20.621082705047 & 60.482989673348 & 120.103951531653 & 199.523657856357 \\
$1 / 256$ & 20.620720381598 & 60.475546521030 & 120.067441003649 & 199.411897858243 \\
$1 / 512$ & 20.620629791855 & 60.473685633053 & 120.058313398197 & 199.383960493605 \\
$1 / 1024$ & 20.620607143877 & 60.473220404839 & 120.056031498617 & 199.376976318024 \\
\hline
\end{tabular}

Table 6 The first four eigenvalues for $I=2$ and different $h$ with $a=0$

\begin{tabular}{lllll}
\hline$h$ & $\lambda_{2 h}^{1}$ & $\lambda_{2 h}^{2}$ & $\lambda_{2 h}^{3}$ & $\lambda_{2 h}^{4}$ \\
\hline $1 / 64$ & 33.539386696208 & 83.321688069218 & 152.883872499399 & 242.450993494860 \\
$1 / 128$ & 33.536292906786 & 83.275738174388 & 152.683074749736 & 241.875210146000 \\
$1 / 256$ & 33.535519115145 & 83.264247113685 & 152.632869196580 & 241.731293670130 \\
$1 / 512$ & 33.535325645709 & 83.261374124815 & 152.620317436233 & 241.695316468978 \\
$1 / 1024$ & 33.535277277012 & 83.260655863631 & 152.617179473073 & 241.686322289850 \\
\hline
\end{tabular}

Example 3 We take $c=\frac{1}{2}, a=1, b=2$, and $l=0,1,2$ as our example. The first four eigenvalues for different $l$ and $h$ are listed in Tables 7-9.

We know from Tables 7-9 that the numerical eigenvalues can achieve six-digit accuracy at least for $h \leq \frac{1}{512}$ for $l=0,1,2$. Similarly, we select the solutions with $h=\frac{1}{1024}$ as the reference solutions. In Figs. 7-8, we plot the error of the approximate eigenvalues. We observe from Figs. 7-8 that the numerical eigenvalues are also convergent. 


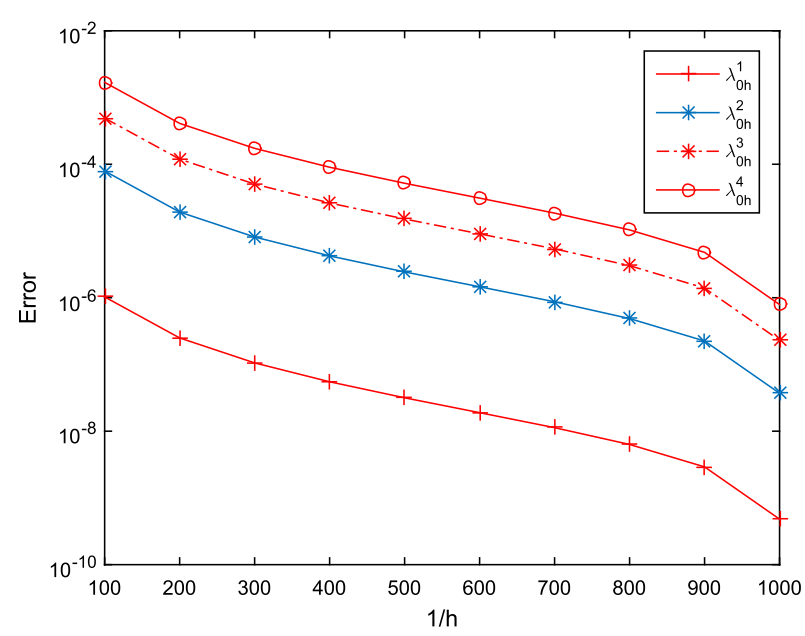

Figure 1 Errors for $I=0$

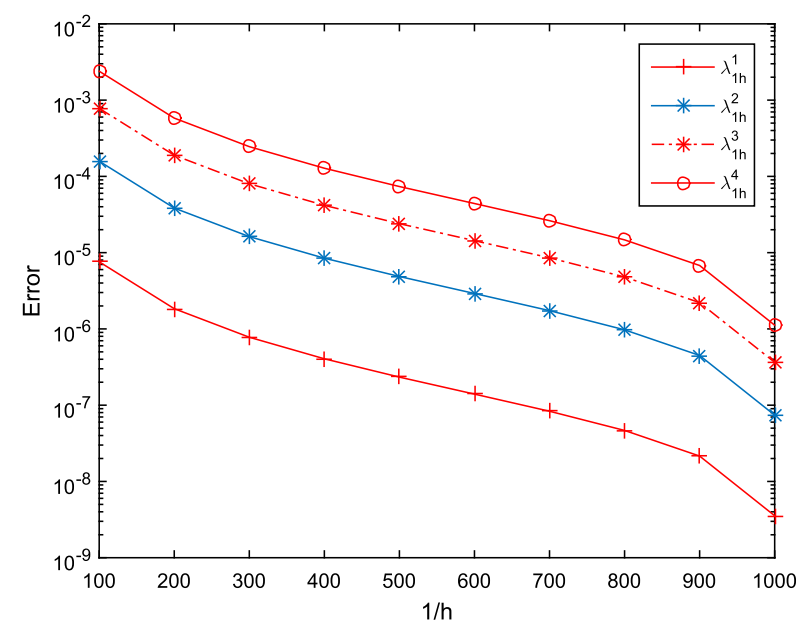

Figure 2 Errors for $I=1$

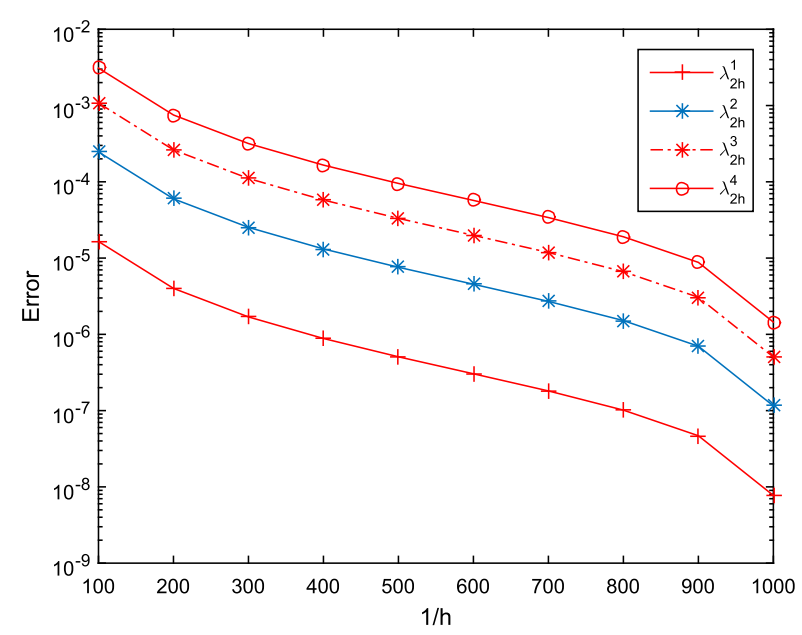

Figure 3 Errors for $I=2$ 


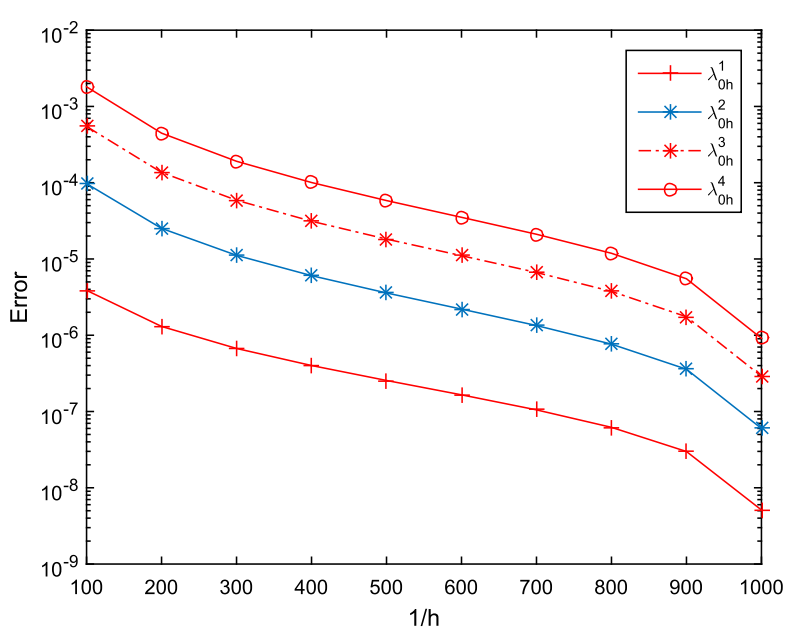

Figure 4 Errors for $I=0$

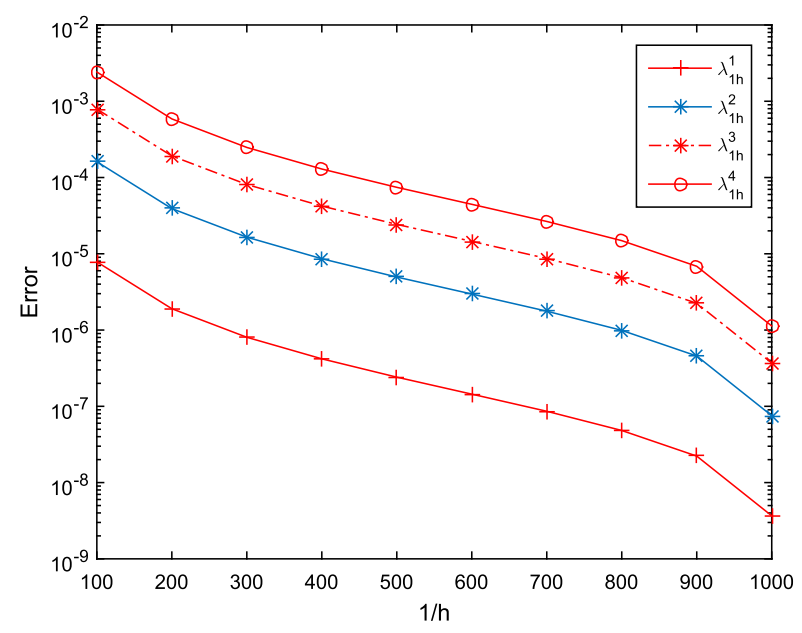

Figure 5 Errors for $I=1$

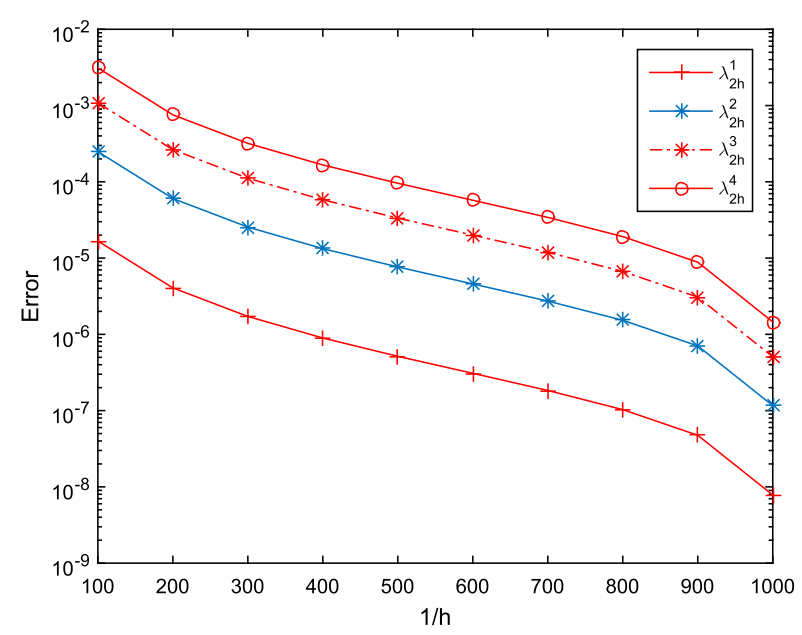

Figure 6 Errors for $I=2$ 
Table $7 a>0$, the four eigenvalues with $/=0$ and $h$

\begin{tabular}{lllll}
\hline$h$ & $\lambda_{0 h}^{1}$ & $\lambda_{0 h}^{2}$ & $\lambda_{0 h}^{3}$ & $\lambda_{0 h}^{4}$ \\
\hline $1 / 64$ & 9.988012930043 & 39.633375167001 & 89.112712061030 & 158.549222565147 \\
$1 / 128$ & 9.986375926619 & 39.608980395076 & 88.990846603507 & 158.165675283969 \\
$1 / 256$ & 9.985966717519 & 39.602883767284 & 88.960402033368 & 158.069907148956 \\
$1 / 512$ & 9.985864417864 & 39.601359739506 & 88.952792255536 & 158.045972559582 \\
$1 / 1024$ & 9.985838843186 & 39.600978740678 & 88.950889896467 & 158.039989377952 \\
\hline
\end{tabular}

Table $8 a>0$, the four eigenvalues with $/=1$ and $h$

\begin{tabular}{lllll}
\hline$h$ & $\lambda_{1 h}^{1}$ & $\lambda_{1 h}^{2}$ & $\lambda_{1 h}^{3}$ & $\lambda_{1 h}^{4}$ \\
\hline $1 / 64$ & 10.915762724023 & 40.613236009777 & 90.103550633775 & 159.544012021946 \\
$1 / 128$ & 10.914153283961 & 40.588873995812 & 89.981719322438 & 159.160499469372 \\
$1 / 256$ & 10.913750964537 & 40.582785551208 & 89.951283273667 & 159.064739988704 \\
$1 / 512$ & 10.913650387204 & 40.581263568823 & 89.943675625219 & 159.040807561191 \\
$1 / 1024$ & 10.913625243058 & 40.580883081317 & 89.941773798436 & 159.034824919945 \\
\hline
\end{tabular}

Table $9 a>0$, the four eigenvalues with $/=2$ and $h$

\begin{tabular}{lllll}
\hline$h$ & $\lambda_{2 h}^{1}$ & $\lambda_{2 h}^{2}$ & $\lambda_{2 h}^{3}$ & $\lambda_{2 h}^{4}$ \\
\hline $1 / 64$ & 12.7606082907083 & 42.5751685615016 & 92.0871194476254 & 161.5348743615320 \\
$1 / 128$ & 12.7590444146313 & 42.5508636384775 & 91.9653485323629 & 161.1514235895622 \\
$1 / 256$ & 12.7586534825387 & 42.5447894481584 & 91.9349275389073 & 161.0556794745995 \\
$1 / 512$ & 12.7585557518340 & 42.5432710282045 & 91.9273236515782 & 161.0317508835969 \\
$1 / 1024$ & 12.7585313192603 & 42.5428914311462 & 91.9254227648108 & 161.0257692010616 \\
\hline
\end{tabular}

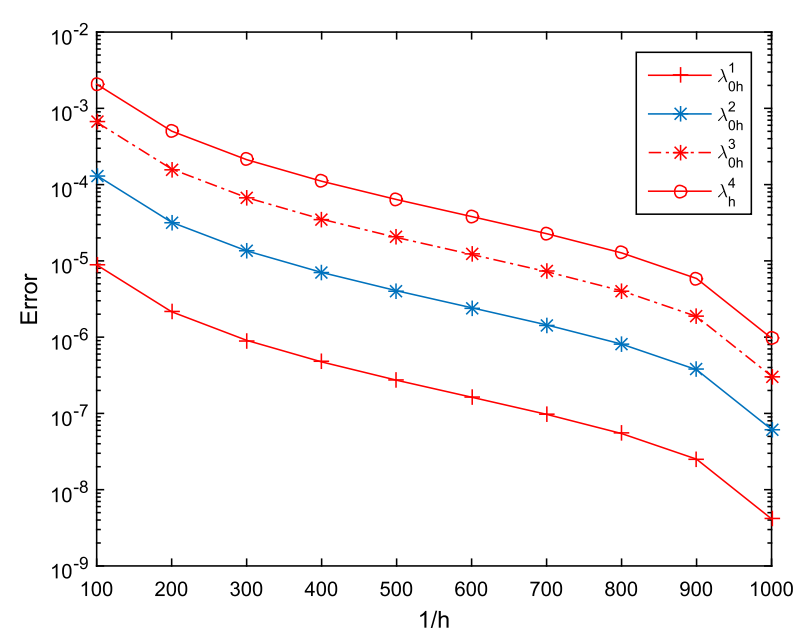

Figure 7 Errors for $l=2$

\section{Conclusions}

In this work, an efficient finite element method is constructed to solve the Schrödinger eigenvalue problem with the IS potential on a spherical domain. By using spherical coordinate transformation and variable separation technique, we reduce the original problem into a series of equivalent and independent eigenvalue problems, which not only overcomes the difficulty brought by the singular coefficient, but also reduces the degrees of freedom greatly by dimension reduction. Thus we can spend less computing time and memory capacity to obtain high-precision numerical solutions. Numerical results show 

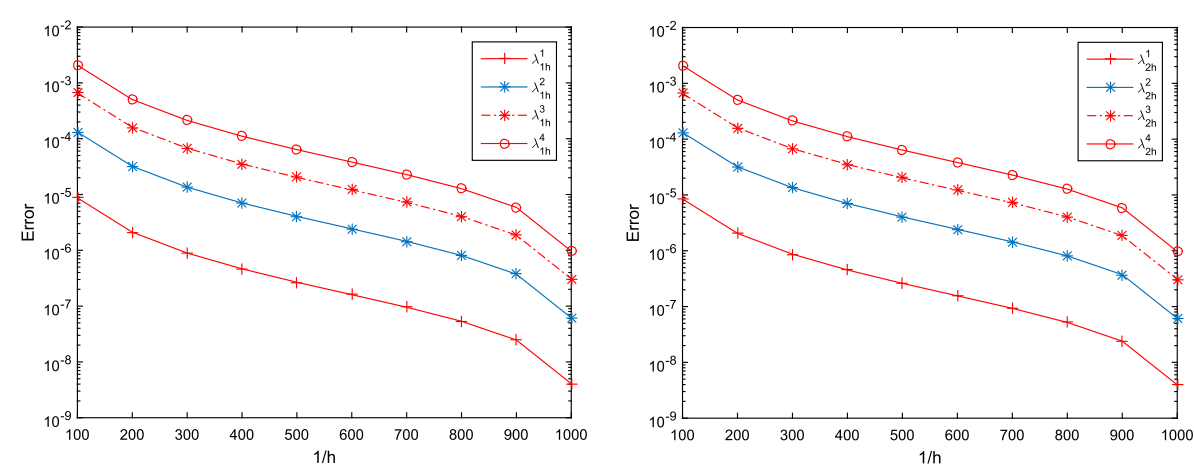

Figure 8 Errors for $I=1$ (left) and $l=2$ (right)

that our algorithm is very effective. We believe that this method can be extended to more complex practical problems.

\section{Acknowledgements}

The authors will thank editors and reviewers for their help.

\section{Funding}

The work of Junying Cao was supported by NSFC (Grant Nos. 11901135, 11961009) and Foundation of Guizhou Science and Technology Department (Grant No. [2020]1Y015). Yubing Sui was supported by Humanities and Social Science Research Projects of Ministry of Education of the People's Republic of China (No. 18YJC790142). Donghao Zhang was supported by Humanities and Social Science Research Projects of Ministry of Education of the People's Republic of China (No. 19YJC790180).

\section{Availability of data and materials}

Data sharing not applicable to this paper as no datasets were generated or analyzed during the current study.

\section{Ethics approval and consent to participate}

Not applicable.

\section{Competing interests}

The authors declare that they have no competing interests.

\section{Consent for publication}

We agree.

\section{Authors' contributions}

YS and ZD carried out an efficient numerical approach to the eigenvalue problem of Schrödinger equation. JC helped to draft the manuscript. JZ is responsible for numerical experiments and proofs. All authors read and approved the final manuscript.

\section{Author details}

${ }^{1}$ College of Economics, Shenzhen University, 518060 Shenzhen, China. ${ }^{2}$ School of Insurance, Southwestern University of Finance and Economics, 610074, Chendu, China. ${ }^{3}$ School of Data Science and Information Engineering, Guizhou Minzu University, 550025 Guiyang, China. ${ }^{4}$ Guizhou Key Laboratory of Big Data Statistics Analysis, Guizhou University of Finance and Economics, 550025 Guiyang, China.

\section{Publisher's Note}

Springer Nature remains neutral with regard to jurisdictional claims in published maps and institutional affiliations.

Received: 17 July 2020 Accepted: 6 October 2020 Published online: 17 October 2020

\section{References}

1. Felli, V., Marchini, E., Terracini, S.: On the behavior of solutions to Schrödinger equations with dipole type potentials near the singularity. Discrete Contin. Dyn. Syst. 21, 91-119 (2008)

2. Fournais, S., Hoffmann-Ostenhof, M., Hoffmann-Ostenhof, T., Østergaard Sørensen, T.: Analytic structure of solutions to multiconfiguration equations. J. Phys. A 42, 315208 (2009)

3. Moroz, S., Schmidt, R.: Nonrelativistic inverse square potential, scale anomaly, and complex extension. Ann. Phys. 325, 491-513 (2010)

4. Wu, H., Sprung, D.W.L.: Inverse-square potential and the quantum vortex. Phys. Rev. A 49, 4305-4311 (1994) 
5. Case, K.M.: Singular potentials. Phys. Rev. 80, 797-806 (1950)

6. Frank, W.M., Land, D.J., Spector, R.M.: Singular potentials. Rev. Mod. Phys. 43, 36-98 (1971)

7. Cao, D., Han, P.: Solutions to critical elliptic equations with multi-singular inverse square potentials. J. Differ. Equ. 224, 332-372 (2006)

8. Felli, V., Marchini, E.M., Terracini, S.: On Schrödinger operators with multipolar inverse-square potentials. J. Differ. Equ. 250, 265-316 (2007)

9. Felli, V., Terracini, S.: Elliptic equations with multi-singular inverse-square potentials and critical nonlinearity. Commun. Partial Differ. Equ. 31, 469-495 (2006)

10. Kalf, H., Schmincke, U.W., Walter, J., Wüst, R.: On the spectral theory of Schrödinger and Dirac operators with strongly singular potentials. In: Spectral Theory and Differential Equations. Comm. Lecture Notes in Math., vol. 448, pp. 182-226. Springer, Berlin (1975)

11. Li, H., Ovall, J.S.: A posteriori eigenvalue error estimation for the Schrödinger operator with the inverse square potential. Discrete Contin. Dyn. Syst., Ser. B 20, 1377-1391 (2015)

12. Ghanbari, B., Atangana, A.: Some new edge detecting techniques based on fractional derivatives with non-local and non-singular kernels. Adv. Differ. Equ. 2020, 435 (2020)

13. Rahman, G., Nisar, K.S., Ghanbari, B., Abdeljawad, T.: On generalized fractional integral inequalities for the monotone weighted Chebyshev functionals. Adv. Differ. Equ. 2020, 368 (2020)

14. Ghanbari, B., Nisar, K.S., Ghanbari, B., Aldhaifallah, M.: Abundant solitary wave solutions to an extended nonlinear Schrödinger's equation with conformable derivative using an efficient integration method. Adv. Differ. Equ. 2020 $328(2020)$

15. Ghanbari, B., Rada, L., Chen, K.: A restarted iterative homotopy analysis method for two nonlinear models from image processing. Int. J. Comput. Math. 91, 661-687 (2014)

16. Khater, M.M.A., Khater, C., et al.: Analytical, semi-analytical, and numerical solutions for the Cahn-Allen equation. Adv. Differ. Equ. 2020, 9 (2020)

17. Khater, M.M.A., Khater, C., et al.: On the numerical investigation of the interaction in plasma between frequency of waves. Results Phys. 18, 103317 (2020)

18. Khater, M.M.A., Khater, C., et al.: Abundant analytical and numerical solutions of the fractional microbiological densities model in bacteria cell as a result of diffusion mechanisms. Chaos Solitons Fractals 136, 109824 (2020)

19. Li, J., Attia, R.A.M., Khater, M.M.A., Lu, D.: The new structure of analytical and semi-analytical solutions of the longitudinal plasma wave equation in a magneto-electro-elastic circular rod. Mod. Phys. Lett. B 12, 2050123 (2020)

20. Khater, M.M.A., Attia, R.A.M., Lu, D.: Computational and numerical simulations for the nonlinear fractional Kolmogorov-Petrovskii-Piskunov (FKPP) equation. Phys. Scr. 95, 055213 (2020)

21. Munusamy, K., Ravichandran, C., Nisar, K.S., Ghanbari, B.: Existence of solutions for some functional integro-differential equations with nonlocal conditions. Math. Methods Appl. Sci. 328 (2020). https://doi.org/10.1002/mma.6698

22. Ghanbari, B., Yusuf, A., Inc, M., Baleanu, D.: The new exact solitary wave solutions and stability analysis for the $(2+1)$-dimensional Zakharov-Kuznetsov equation. Adv. Differ. Equ. 2019, 49 (2019)

23. Srivastava, H.M., Günerhan, H., Ghanbari, B.: Exact traveling wave solutions for resonance nonlinear Schrödinger equation with intermodal dispersions and the Kerr law nonlinearity. Math. Methods Appl. Sci. (2020). https://doi.org/10.1002/mma.5827

24. Christiansen, P.L., Muto, V., Rionero, S.: Solitary wave solutions to a system of Boussinesq-like equations. Chaos Solitons Fractals 1, 45-50 (1992)

25. Khater, M.M.A., Attia, R.A.M., Abdel-Aty, A.H., et al.: Analytical and semi-analytical ample solutions of the higher-order nonlinear Schrödinger equation with the non-Kerr nonlinear term. Results Phys. 12, 103000 (2020)

26. Khater, M.M.A., Attia, R.A.M., Abdel-Aty, A.H., et al.: Analytical and numerical solutions for the current and voltage model on an electrical transmission line with time and distance. Phys. Scr. 95, 055206 (2020)

27. Felli, V., Ferrero, A., Terracini, S.: Asymptotic behavior of solutions to Schrödinger equations near an isolated singularity of the electromagnetic potential. J. Eur. Math. Soc. 13, 119-174 (2011)

28. Hunsicker, E., Li, H., Nistor, V., Ville, U.: Analysis of Schrödinger operators with inverse square potentials I: regularity results in 3D. Bull. Math. Soc. Sci. Math. Roum. 55, 157-178 (2012)

29. Li, H., Nistor, V.: Analysis of a modified Schrödinger operator in 2D: regularity, index, and FEM. J. Comput. Appl. Math. 224, 320-338 (2009)

30. Li, H., Zhang, Z: Efficient spectral and spectral element methods for eigenvalue problems of Schrödinger equations with an inverse square potential. SIAM J. Sci. Comput. 39(1), A114-A140 (2017)

31. Li, H., Ovall, J.S.: A posteriori estimation of hierarchical type for the Schrödinger operator with the inverse square potential on graded meshes. Numer. Math. 128, 707-740 (2014)

32. Reddien, G.W.: Finite-difference approximations to singular Sturm-Liouville eigenvalue problems. Math. Comput. 30, 278-282 (1976)

33. Ma, L., Shen, J., Wang, L.L.: Spectral approximation of time-harmonic Maxwell equations in three-dimensional exterior domains. Int. J. Numer. Anal. Model. 12, 1-18 (2015)

34. Babuška, I., Osborn, J.: Eigenvalue Problems. Handbook of Numerical Analysis, vol. II, pp. 640-787. Elsevier, Amsterdam (1991) 